\title{
Perceived Personal, Social and Environmental factors that lead to Obesity among Female Students in Menoufia University
}

\author{
Dr. Amal Attia K.Hussein \\ Assistant Professor of Community Health Nursing Department \\ Faculty of Nursing - Menoufia University \\ Dr Amal Amien El-Sheikh \\ Lecturer of Adult Health Nursing Department \\ Faculty of Nursing - Menoufia University \\ Elham Sobhy Mostafa Elzyen \\ Clinical Instructor of Community Health Nursing Department \\ Faculty of Nursing - Menoufia University
}

\begin{abstract}
Obesity is a metabolic disease in which adipose tissue comprises a greater than normal proportion of body tissue. An individual can be considered to be obese when the amount of body fat increases beyond the point where their health begins to deteriorate. Extreme obesity is often associated with a shortened life expectancy .The study' aim: was to determine the perceived personal, social and environmental factors that lead to obesity among university students. Method and Subjects: A cross sectional design was used. Sample: of 1000 students of the first, second, third and fourth year. Setting: Faculty of Art and Nursing in Shebin Elkom- Menoufia University were chosen by simple random sample. Tools: Three tools were utilized for data collection.I An interview questionnaire including; Sociodemographic data and medical history of the student and her family, Measure some aspects of student's life style as physical activity and eating habits, Students knowledge about obesity II perceived factors to weight maintenance questionnaire III Biophsiological measurement. Results: age of the studied sample ranged from 18-24 years old. $40.8 \%$ of the students were overweight, and $1.3 \%$ of students were underweight. The result showed lack of time, no safe environment and lack of access to the places for physical activity. There was statistically significant difference between normal vs overweight and obese students regarding support from spouse or family members for physical activity Conclusion: lack of time, no safe environment and lack of access to the places for exercising and unhealthy diet lead to obesity. Recommendations: Future research should study further psychosocial stressors
\end{abstract}


and educational environmental stressors that influence food selection, preparation and intake also attitude towards health fitness and activity among female students in Menoufia University.

Key words: Obesity, Personal, Social and Environmental factors.

\section{Introduction}

Obesity is considered the most important malnutrition status allover the world that defined as having more body fat than the healthy standard which increases the health hazards . Obesity is a common public health problem of the 21 st century because it is the leading cause of death worldwide, with increasing prevalence in adults and children. It has reached an epidemic proportion globally, with more than one billion adults' world wide being obese (Barness et al., 2007).

In 2006 the World Health Organization (WHO) estimated that there were approximately 1.1 billion overweight (body mass index: $\mathrm{BMI} \geq 25 \mathrm{~kg} / \mathrm{m} 2$ ) adults worldwide and a further 312 million adults that are obese $(B M I \geq 30 \mathrm{~kg} / \mathrm{m} 2)$ (Hossain et al., 2007) with South Africa also not being spared in this global increase (Puoane et al., 2002). Egypt is confronted to the double burden of over-nutrition. Overweight and obesity are reaching alarming levels, starting at young age. Some $28 \%$ of women aged 15-59 years are overweight and 40\% are obese (CAPMAS, 2011).

One group at particular risk of weight gain and the development of obesity is young women. Obesity is most commonly caused by a combination of excessive food energy intake, lack of physical activity and genetic susceptibility, although a few cases are caused primarily by genes, endocrine disorders, medications or psychiatric illness (Adams, 2006).

Young women are at high risk of weight gain but there has been limited knowledge on weight management in these groups. Young women are more likely to gain weight than young men. Each generation of young women also supersedes the previous generation in weight gain. Obesity in young women before they enter motherhood is a pivotal point in reversing the trends of obesity in future generations (WHO, 2000). Risk of weight gain and the development of obesity places young women at increased risk of chronic medical conditions and diseases, such as hypertension, non-insulin- 
dependent diabetes mellitus or type 2 diabetes, cardiovascular disease, respiratory diseases, knee, hip and back problems and some cancers as well as early death. Over weight and obesity can affect the quality of life in young women by impairing their reproductive health. Obese young women are at greater risk of irregular periods, infertility and polycystic ovaries syndrome (Kopelman, 2007).

Over weight or obesity in early adulthood can have significant social implication for young women. The effect of obesity on mental health of young women also contribute to attention, anxiety and depression which the leads to increase burden of disease and injury. Over weight or obese young women are less likely to report satisfaction with work or study, family relationships or social activities (WHO, 2000).

More frequently women reported tiredness and lack of time as perceived significant social barriers to healthy habits than do men, and this may be partly attributable to their domestic situation (Brownson, 2001). In addition, young women are more likely than older women to experience particular life events such as starting work, leaving family homes to marry and becoming mothers. Also, lack of information, skills to plan and prepare healthy diet, easy availability of fast food, lack of motivation and time to eat healthy food, lack of family, friends' encouragement and support may influence their physical activity and dietary habits (Kear, 2007).

The perceived barriers to increasing physical activity and improving diet may vary according to social and personal circumstances. For example, having children is likely to impact on a women's ability to adopt personal healthy habits. In addition, persons of lower socioeconomic status may have poorer access to parks, walking and gym equipment than those of higher socioeconomic status (Ball, 2004).

Behavioral weight loss interventions that focus on diet and $\backslash$ or exercise are considered the most effective management of over weight. Giving health instruction about weight management, physical activity, and food intake, describing in detail the nature of the interventions and the different ways in which family members could be involved will have positive effect (Whitlock, 2008). Being physically active improves mental and musculoskeletal health and reduces other risk factors such as overweight, high blood pressure and high blood cholesterol (Sproston et al., 2003). 
Nurses can promote healthy lifestyle patterns that reduce the risks of being overweight or obese. For example, breastfeeding, physical activity, regular meals, and nutrition and weight counseling are all areas where nurses may help to reduce the risk of obesity .Nurses constitute a political force in health policy, because they are situated in a wide range of settings. Thus, nurses are on the frontline of obesity prevention and health promotion (International Council of Nurses, 2009).

\section{Aim of the Study}

\section{The aim of this study is to:}

1- Determine the perceived personal, social and environmental factors that lead to obesity among female university students.

\section{Research Question:}

- What are the personal, social and environmental factors that lead to obesity among female university students?

\section{Subjects and Methods}

\section{Research design:}

Descriptive design was utilized to achieve the aim of the study.

\section{Research Setting:}

The study was conducted at two faculties in Menoufia University, Egypt (one is practical and the other is theoretical). Menofia University consists of 11 practical faculties and 8 theoretical faculties. Two faculties were selected randomly from a container containing names of practical faculties and the other one containing names of theoretical faculties. The resulting selection of faculties was faculty of Arts and faculty of Nursing. Data were collected at the Students Affairs Departments to ensure accessibility of students or in section room which was prepared before to gather students for filling the questionnaires.

\section{Subjects:}

The total number of female students in faculty of Arts was 5342 (147 student in first grade, 256 student in second grade, 2565 student in third grade, 2374 student in fourth grade) while the total number of students in faculty of Nursing is 636 (147 student in first grade, 114 student in second grade, 143 student in third grade, 236 student in fourth grade).

All students who agreed to participate from the first, second, third, and fourth grade of both faculties were included in the study. The total number of the sample was 1000 subjects (800 students in faculty of Arts and 200 students in faculty of Nursing),

\section{Power analysis:}

The total number of female students in faculty of Arts was 5342. With power $90 \%$ the sample size were 800 students. So, we 
collect our data for 800 students. while the total number of students in faculty of Nursing is 636 . With power $90 \%$ the sample size were 200 students. So, we collect our data for 200 students

\section{Exclusion criteria:}

- Pregnant females.

- Students in hormone therapy.

- Presence of chronic disease as diabetes.

- Students in special dietary or medical regimen.

- Students on cortisone therapy.

\section{Tools of the study:}

Three tools were utilized by the researcher for data collection.

Tool I: questionnaire(female student's knowledge regarding life style structure questionnaire)

A-An interview questionnaire was constructed by the researcher depending on a review of related literature to assess some aspects of student's life style as physical activity and eating habits and students knowledge about obesity. It included three parts.

\section{Part one: Sociodemographic and medical} data

It included data about sociodemographic characteristics as student's age, academic year, martial status, residence, number of family members. Sociodemographic data related to parents as level of education, occupation, type of living, income and family history of disease.

Part two: Questions to measure some aspects of student's life style as physical activity and eating habits

\section{A- Physical activities}

It comprised 12 questions about physical activity habits such as type and amount of activity, sleeping hours, TV watching period and computer working period.

\section{B -Information about dietary Practice:}

It was developed by the researcher to gather information about dietary practices of the students and 24 hours food recall. It consisted of two sections:-

Section one: Dietary practice.

It consisted of 19 questions about number of meals /day, methods of cooking food, snacking, kind of daily fluid intake, etc.

Section two: The 24 hours food recall sheet that was adopted from the National Nutrient Database for Standard Reference, (2000).

It was used to recall nutritional elements of the 24 hours recall of dietary intake for the last 3 days. The student was asked to report all consumed foods on the previous three days including detailed description of all food beverages and supplements with portions size in household measurements. 
Part three: Students knowledge about obesity

It has 4 open ended questions related to student's knowledge about obesity (definition, causes, complication and guidelines which can be followed to control obesity). Each question was given 1 mark if the subject reported correct answer, 0 if the subject reported wrong answer.

Tool II: Perceived factors to weight maintenance questionnaire developed by Andajani-Sutjahjo., etal,( 2004).It contains 18 questions about perceived factors leading to obesity. It consisted of 3 sections; personal, environmental and social factors on a 2 level scale (Yes/ No). The scores were:

Items scores

Yes 1

No 0

Personal factors: It comprises 10 questions related to physical activity and healthy eating. For each question, students were asked about access to information; motivation; enjoyment and skills to plan, prepare or cook healthy foods, weight is appropriate for their height and age, weigh yourself regularly, satisfied with your body weight and problems with your body weight.

The World Health Organization (WHO) classified obesity according to BMI as: 


\begin{tabular}{|l|l|}
\hline Classification & BMI $(\mathbf{k g} / \mathbf{m} 2)$ \\
\hline Underweight & Less than 18.5 \\
\hline Normal range & $18.5-24.9$ \\
\hline Overweight & $25-29.9$ \\
\hline Obese I & $30-34.9$ \\
\hline Obese II & $35-39.9$ \\
\hline Obese III & Greater than or equal to 40.0 \\
\hline
\end{tabular}

Modifications were done accordingly to

World Health Organization. (2000). The

Asia-Pacific perspective: redefining obesity

and its treatment. Health Communications

Australia Pty Ltd. Available at:-

http://www.idi.org.au/obesity-report.htm.

Retrieved on: - (January 6, 2013).

\section{II-Method}

An official permission was obtained from the dean of Faculty of Nursing \& dean of Art Faculty \&Vice dean of two faculties of education and Students' Affairs and directed to the authorized personnel at the various settings for data collection.

\section{Tools development: -}

The first tool was developed by the

researcher after extensive review of the relevant literature.

\section{Validity of the tools:}

All tools were tested for its content validity by three experts in the field of Medical Surgical Nursing, and three experts in the field of Community Health Nursing, Faculty of Nursing, Menoufia University. ascertain relevance and completeness

\section{Reliability of the tools:}

Reliability was measured by test -re-test for measuring the internal consistency of the tool. The tool was administered to the same subjects (twenty students) under similar conditions two times, fifteen days apart. The subject's answers from the repeated testing were compared (Test- Re -test reliability) (r $=84)$.

\section{Ethical consideration:}

Informed consent was obtained after the purpose of the study was explained to them. Also they were assured that, the information would remain confidential .The researcher emphasized that participation in the study is entirely voluntary and anonymity of the students were assured through data coding and they could withdraw at any stage of the research.

\section{Pilot study:-}

Before starting data collection, pilot study was conducted in order to test tools' clarity 
and applicability. The sample recruited 10 students for the pilot study according to the criteria of selection. Necessary modifications were done accordingly. The pilot subjects were excluded from the total sample of the study to assure stability of the result.

\section{Data collection:-}

- After the acceptance for conducting this study by the Faculty of NursingMenoufia University, Board for Higher Education; an official permission was obtained from Menoufia University Vice dean of Education and Students' Affairs and deans of the two faculties. Students were chosen with consideration of the exclusion criteria and a self-administration technique was used to collect data.

- Data collection was carried out in the period from March 2012 to October 2012.The researcher collected the data in the class room during the afternoon at two days/ week from $12 \mathrm{PM}$ to 4:15 PM. The subjects were divided into 40 groups; each of them consisted of 25 students.

- The researcher introducing herself to participant.
- Assessment of the nutritional status of students was done using dietary practice tool by collecting dietary history, dietary recall. For each food, calculations were made for the contribution of food for protein, carbohydrates, fats and calories. Estimation of adequacy of nutrient intake was done based on data for Recommended Individual Dietary Intakes. Assessment factors lead to obesity, consisted of 3 sections; personal, environmental and social factors. Then biophysiological measurement was carried out as follows:

\section{a- Height measurement}

The plastic tape was pasted on wall of the classroom. Then student was asked to remove his shoes, and other bulky clothing. Then the student was asked to stand erect beside the wall, with hands at sides, knees or thighs together and his/ her weight distributed on both feet. Then read the height to the nearest value $0.5 \mathrm{~cm}$.

\section{b- weight measurement}

A platform scale was used. The student was asked to remove bulky clothes and shoes. Place the scale on the zero value before the student steps on the scale. The student is 
asked to stand with both feet on the center of the platform scale, and read the measurement to the nearest 0.5 kilogram. Then body mass index ((BMI) is calculated as follows:

Body Mass Index $=$ weight $(\mathrm{kg}) /$ height $(\mathrm{m})^{2}$.

The average time spent for collecting data from each student was approximately 10 to 15 minutes for questionnaire sheet and 10 minutes for body mass index measurement. The researchers distributed a booklet written in a simple language and have pictures to help participants to select healthy balanced diets and some simple exercises to follow and to remind the participants about healthy diet and exercises to follow. This booklet was developed by the researcher and was tested for its content validity by experts in the field of nutrition.

\section{Statistical analysis:}

The collected data were organized, tabulated and statistically analyzed using SPSS software statistical computer package version 16. For qualitative data, comparison between two groups and more was done using Chi-square test $\left(\chi^{2}\right)$. For comparison between means of two groups, parametric analysis (t-test) and non-parametric analysis ( $Z$ value of Mann-Whitney $U$ test) were used. For comparison between more than two means of parametric analysis, the $\mathrm{F}$ value of analysis of variance (ANOVA) was calculated. For comparison between more than two means of non-parametric analysis, the $\chi^{2}$ value of Kruskal-Wallis Test was calculated. Significance was adopted at $\mathrm{p}<0.05$ for interpretation of results of tests of significance (Petrie, 2005).

\section{Results}

Table 1 displayed Socio-demographic data of the studied students. The result showed that the mean age of the studied subject was 19.95 \pm 1.05 . Regarding academic year near to half of them $(47.0 \%)$ was grade IV. For martial status, most of them were single $(93.8 \%)$. It was found that the majority (77.1\%) were from rural areas. Regarding type of housing the majority live in nuclear family. As for monthly income the majority reported having enough income.

There was no statistical significant difference between the two faculties' students except for academic year, mother's occupation, and number of family members $\mathrm{p}=(0.0001,0.010,0.0001)$ respectively.

Table 2 showed distribution of medical history of the family among the studied student's (Normal vs overweight \& obese). About one-third of overweight \& obese students had family history of overweight.

Table 3 revealed that the mean weight for faculty of Arts students was $66.89 \pm 12.00 \mathrm{Kg}$ 
and $65.27 \pm 10.45 \mathrm{Kg}$ for faculty of nursing students. Also, the mean height for faculty of Arts students' was 158.30 $\pm 9.59 \mathrm{Cm}$ and $159.01 \pm 10.86 \mathrm{Cm}$ for faculty of nursing students. There was statistically significant difference between two faculties' students regarding body mass index where $\mathrm{p}=0.027$.

Figure 1 showed distribution of knowledge among normal vs overweight \& obese. Regarding knowledge about definition of obesity $51.9 \%$, of normal body weight group knew the correct answer compared to $46.9 \%$ of overweight and obese students.

Also, the majority of normal body weight students $(71.3 \%)$ and (64.3\%) of overweight and obese students know the correct answer about causes of obesity.

Regarding complication of obesity, the majority of normal body weight students (78.3\%) and (73.3\%) of overweight and obese students knew the correct answer.

As regards to guidelines which can be followed to control obesity, over three fourths $(76.1 \%)$ of normal body weight students and (75.0\%) of overweight and obese students knew the correct answer.

Figure 2 it is clear from the figure that $60.5 \%$ of normal weight students and $63.2 \%$ of overweight\& obese students reported that thy practicing physical exercise
Table 4 showed that there was statistically significant difference between normal vs overweight and obese students regarding number of meals per day $(\mathrm{p}=0.013)$, kind of fat used $(\mathrm{p}=0.006)$, kind of daily fluid $(\mathrm{p}=$ 0.0001).

Table 5 showed that there was statistically significant difference between normal, overweight \& obese students regarding all items of macronutrients. With higher mean values of all items among obese students than overweight students which was also higher than normal.

Table 6 showed that $51.3 \%$ of normal body weight students compared to $48.7 \%$ of overweight and obese students had information about the importance of physical activity with statistically significant difference between normal vs overweight and obese students $(\mathrm{p}=0.0001)$. Also, 65.2\% and $67.3 \%$ of normal body weight vs overweight and obese students respectively reported enjoying practicing physical activity. The majority of normal vs overweight and obese students $(84.6 \%$ and $80.6 \%$ ) respectively were motivated to follow a healthy diet instruction and had the skill required to prepare healthy foods (80.6\% and $80.1 \%)$ respectively.

There was statistically significant difference between normal vs overweight and obese 
students regarding source of information $\mathrm{p}=$ 0.002, think that their weight was appropriate for their height and age, satisfied with body weight $\mathrm{p}=0.0001$ and problems with body weight where $\mathrm{p}=0.012$.

Table 7 showed no statistical significant difference between normal vs overweight and obese students in all perceived environmental factors.

Table 8 showed that two thirds of normal weight students reported that they don't have spouse or family members support for physical activity (70.8\%), lack of costeffective places $(73.0 \%), 58.4 \%$ of students have support from spouse or family members to follow a healthy diet and $51.4 \%$ reported that healthy food was no expensive. On the other hand, near to two thirds of overweight \& obese students reported lack of spouse or family members support for physical activity, lack of costeffective places $(70.5 \%)$ and $54.1 \%$ reported that healthy food were expensive.

There was statistically significant difference

Table (1): Percentage distribution of socio-demographic data among the studied students (Faculty of Arts and Faculty of nursing students) $(n=1000)$.

\begin{tabular}{|c|c|c|c|c|c|c|c|c|}
\hline \multirow[t]{2}{*}{$\begin{array}{c}\text { Socio-demographic } \\
\text { data }\end{array}$} & \multicolumn{2}{|c|}{$\begin{array}{c}\text { Faculty of } \\
\text { Arts } \\
\text { students } \\
(\mathbf{n}=800)\end{array}$} & \multicolumn{2}{|c|}{$\begin{array}{c}\text { Faculty of } \\
\text { Nursing } \\
\text { students } \\
(n=200)\end{array}$} & \multirow{2}{*}{\multicolumn{2}{|c|}{ Total }} & \multirow[t]{2}{*}{$\chi^{2}$} & \multirow[t]{2}{*}{$\mathbf{P}$} \\
\hline & No & $\%$ & No & $\%$ & & & & \\
\hline
\end{tabular}




\begin{tabular}{|c|c|c|c|c|c|c|c|c|}
\hline \multirow{2}{*}{$\begin{array}{l}\text { Range } \\
\text { Mean } \pm \text { SD } \\
\text { t-test } \\
\mathbf{P}\end{array}$} & \multicolumn{2}{|c|}{$\begin{array}{c}18-24 \\
20.02 \pm 1.02 \\
\end{array}$} & \multicolumn{2}{|c|}{$\begin{array}{c}18-23 \\
19.68 \pm 1.11 \\
\end{array}$} & \multicolumn{2}{|c|}{$\begin{array}{c}18-24 \\
19.95 \pm 1.05 \\
\end{array}$} & & \\
\hline & \multicolumn{4}{|c|}{$\begin{array}{c}4.103 \\
0.0001^{*} \\
\end{array}$} & & & & \\
\hline $\begin{array}{l}\text {-Academic year: } \\
1^{\text {st }}\end{array}$ & 49 & 6.1 & 49 & 24.5 & 98 & 9.8 & 157.52 & 0.0001 \\
\hline $2^{\text {nd }}$ & 38 & 4.8 & 47 & 23.5 & 86 & 8.5 & & \\
\hline $3^{\text {rd }}$ & 320 & 40.0 & 27 & 13.5 & 347 & 34.7 & & \\
\hline $4^{\text {th }}$ & 393 & 49.1 & 77 & 38.5 & 470 & 47.0 & & \\
\hline $\begin{array}{l}\text {-Marital status: } \\
\text { Single } \\
\text { Married }\end{array}$ & $\begin{array}{c}746 \\
54\end{array}$ & $\begin{array}{c}93.3 \\
68\end{array}$ & $\begin{array}{c}192 \\
8\end{array}$ & $\begin{array}{c}96.0 \\
40\end{array}$ & $\begin{array}{c}938 \\
62\end{array}$ & $\begin{array}{l}93.8 \\
62\end{array}$ & 2.081 & 0.149 \\
\hline $\begin{array}{l}\text { Married } \\
\text { Residence: }\end{array}$ & 54 & 6.8 & 8 & 4.0 & 62 & 6.2 & & \\
\hline $\begin{array}{l}\text { •Residence: } \\
\text { Rural }\end{array}$ & 607 & 75.9 & 164 & 82.0 & 771 & 77.1 & 3.400 & 0.065 \\
\hline Urban & 193 & 24.1 & 36 & 18.0 & 229 & 22.9 & & \\
\hline $\begin{array}{l}\text {-Father's or } \\
\text { husband education } \\
\text { level: }\end{array}$ & & & & & & & & \\
\hline Illiterate & 56 & 7.0 & 10 & 5.0 & 66 & 6.6 & 6.36 & 0.095 \\
\hline Basic & 158 & 19.7 & 27 & 13.5 & 185 & 18.5 & & \\
\hline Secondary & 367 & 45.9 & 97 & 48.5 & 464 & 46.4 & & \\
\hline University educ. & 219 & 27.4 & 66 & 33.3 & 285 & 28.5 & & \\
\hline
\end{tabular}

*Significant $(\mathbf{P}<0.05)$ 
Table (1): Continue.

\begin{tabular}{|c|c|c|c|c|c|c|c|c|}
\hline \multirow[t]{2}{*}{ Socio-demographic data } & \multicolumn{2}{|c|}{ 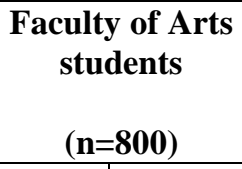 } & \multicolumn{2}{|c|}{$\begin{array}{c}\text { Faculty of } \\
\text { Nursing students } \\
(\mathbf{n}=\mathbf{2 0 0})\end{array}$} & \multicolumn{2}{|c|}{$\begin{array}{c}\text { Total } \\
(\mathrm{n}=1000)\end{array}$} & \multirow[t]{2}{*}{$\chi^{2}$} & \multirow[t]{2}{*}{$\mathbf{P}$} \\
\hline & No & $\%$ & No & $\%$ & No & $\%$ & & \\
\hline $\begin{array}{l}\text {-Mother's education level: } \\
\text { Illiterate } \\
\text { Basic } \\
\text { Secondary } \\
\text { University educ. }\end{array}$ & $\begin{array}{l}157 \\
140 \\
393 \\
110\end{array}$ & $\begin{array}{l}19.6 \\
17.5 \\
49.1 \\
13.8\end{array}$ & $\begin{array}{c}30 \\
32 \\
109 \\
29\end{array}$ & $\begin{array}{l}15.0 \\
16.0 \\
54.5 \\
14.5\end{array}$ & $\begin{array}{l}187 \\
172 \\
502 \\
139\end{array}$ & $\begin{array}{l}18.7 \\
17.2 \\
50.2 \\
13.9\end{array}$ & 3.03 & 0.388 \\
\hline $\begin{array}{l}\text {-Mother's occupation: } \\
\text {-Housewife } \\
\text {-Employee } \\
\text { - Manual work } \\
\text {-Died }\end{array}$ & $\begin{array}{c}514 \\
242 \\
43 \\
1 \\
\end{array}$ & $\begin{array}{c}64.4 \\
30.3 \\
5.4 \\
0.1 \\
\end{array}$ & $\begin{array}{c}124 \\
74 \\
2 \\
0 \\
\end{array}$ & $\begin{array}{c}62.0 \\
37.0 \\
1.0 \\
0 \\
\end{array}$ & $\begin{array}{c}638 \\
316 \\
45 \\
1\end{array}$ & $\begin{array}{c}63.9 \\
31.7 \\
4.5 \\
0.1 \\
\end{array}$ & 9.23 & $0.010^{*}$ \\
\hline $\begin{array}{l}\bullet \text { No of family members: } \\
1-3 \\
4-5 \\
>6 \\
\end{array}$ & $\begin{array}{c}77 \\
447 \\
276 \\
\end{array}$ & $\begin{array}{r}9.6 \\
55.9 \\
34.5 \\
\end{array}$ & $\begin{array}{c}12 \\
76 \\
112 \\
\end{array}$ & $\begin{array}{c}6.0 \\
38.0 \\
56.0 \\
\end{array}$ & $\begin{array}{r}89 \\
523 \\
388 \\
\end{array}$ & $\begin{array}{r}8.9 \\
52.3 \\
38.8 \\
\end{array}$ & 31.199 & $0.0001 *$ \\
\hline $\begin{array}{l}\text {-Type of living: } \\
\text { Nuclear family } \\
\text { Extended family }\end{array}$ & $\begin{array}{l}472 \\
328\end{array}$ & $\begin{array}{l}59.0 \\
41.0\end{array}$ & $\begin{array}{c}126 \\
74\end{array}$ & $\begin{array}{l}63.0 \\
37.0\end{array}$ & $\begin{array}{l}598 \\
402\end{array}$ & $\begin{array}{l}59.8 \\
40.2\end{array}$ & 1.065 & 0.302 \\
\hline -Monthly income : & & & & & & & & \\
\hline Not enough & 153 & 19.1 & 40 & 20.0 & 193 & 19.3 & 3.458 & 0.177 \\
\hline Enough & 559 & 69.9 & 129 & 64.5 & 688 & 68.8 & & \\
\hline More than enough & 88 & 11.0 & 31 & 15.5 & 119 & 11.9 & & \\
\hline
\end{tabular}

*Significant $(\mathbf{P}<\mathbf{0 . 0 5})$

Table (2): Percentage distribution of the reported medical history of the family among the studied students (Normal vs overweight \& obese).

\begin{tabular}{|c|c|c|c|c|c|c|c|c|}
\hline \multirow[t]{2}{*}{ Variables } & \multicolumn{2}{|c|}{$\begin{array}{c}\text { Normal body } \\
\text { weight students } \\
(n=397)\end{array}$} & \multicolumn{2}{|c|}{$\begin{array}{l}\text { Overweight \& } \\
\text { Obese students } \\
\qquad(\mathrm{n}=603)\end{array}$} & \multicolumn{2}{|c|}{ Total students } & \multirow{2}{*}{$\begin{array}{c}\chi^{2} \\
\& \\
\text { Fisher } \\
\text { Exact } \\
\text { test } \\
\end{array}$} & \multirow[t]{2}{*}{$\mathbf{P}$} \\
\hline & No & $\%$ & No & $\%$ & No & $\%$ & & \\
\hline $\begin{array}{l}\text {-Family history of disease } \\
\text {-Overweight }\end{array}$ & 66 & 26.8 & 129 & 34.4 & 195 & 31.4 & 3.467 & 0.063 \\
\hline -Diabetes & 97 & 23.7 & 159 & 24.0 & 239 & 23.9 & 0.428 & 0.514 \\
\hline -Heart disease & 34 & 13.8 & 39 & 10.4 & 73 & 11.7 & 1.555 & 0.212 \\
\hline -Respiratory dis. & 22 & 8.9 & 44 & 11.7 & 66 & 10.6 & 1.197 & 0.274 \\
\hline -Liver \& Gall bladder dis. & 59 & 24.0 & 87 & 23.2 & 146 & 23.5 & 0.036 & 0.849 \\
\hline -Digestive syst. Dis. & 46 & 18.7 & 66 & 17.6 & 112 & 18.0 & 0.099 & 0.753 \\
\hline -Joint \& bone dis. & 106 & 43.1 & 146 & 38.9 & 252 & 40.6 & 0.786 & 0.375 \\
\hline -Urinary system dis. & 26 & 10.6 & 40 & 10.7 & 66 & 10.6 & 0.003 & 0.958 \\
\hline
\end{tabular}

*Significant $(\mathbf{P}<0.05)$

Table (3): Percentage distribution of body weight, height and body mass index (BMI) among the studied students (Faculty of Arts and Faculty of nursing students). 


\begin{tabular}{|c|c|c|c|c|c|}
\hline \multirow[t]{2}{*}{ Variables } & $\begin{array}{c}\text { Faculty of } \\
\text { Arts } \\
\text { students } \\
(\mathbf{n}=\mathbf{8 0 0})\end{array}$ & $\begin{array}{c}\text { Faculty of } \\
\text { Nursing } \\
\text { students } \\
(n=200)\end{array}$ & $\begin{array}{c}\text { Total } \\
(\mathbf{n}=\mathbf{1 0 0 0})\end{array}$ & \multirow[t]{2}{*}{ t-test } & \multirow[t]{2}{*}{$\mathbf{P}$} \\
\hline & $\begin{array}{c}\text { Range } \\
\text { Mean } \pm \text { SD }\end{array}$ & $\begin{array}{c}\text { Range } \\
\text { Mean } \pm S D\end{array}$ & $\begin{array}{c}\text { Range } \\
\text { Mean } \pm \text { SD }\end{array}$ & & \\
\hline -Weight (kg) & $\begin{array}{c}34-166 \\
66.89 \pm 12.00\end{array}$ & $\begin{array}{c}46-105 \\
65.27 \pm 10.45\end{array}$ & $\begin{array}{c}34-166 \\
66.57 \pm 11.72\end{array}$ & 1.754 & 0.080 \\
\hline •Height (cm) & $\begin{array}{c}45-178 \\
158.30 \pm 9.59\end{array}$ & $\begin{array}{c}52-196 \\
159.01 \pm 10.86\end{array}$ & $\begin{array}{c}45-196 \\
158.44 \pm 9.85\end{array}$ & 0.906 & 0.998 \\
\hline $\begin{array}{l}\text {-BMI (Weight } \\
(\mathrm{kg}) / \text { height (meter) }{ }^{2}\end{array}$ & $\begin{array}{l}17.40-48.88 \\
26.49 \pm 4.28\end{array}$ & $\begin{array}{l}17.53-39.55 \\
25.74 \pm 4.23\end{array}$ & $\begin{array}{l}17.40-78.88 \\
26.34 \pm 4.28\end{array}$ & 2.211 & $0.027^{*}$ \\
\hline
\end{tabular}

*Significant $(\mathbf{P}<\mathbf{0 . 0 5})$

Figure (1): Percentage distribution of correct information about overweight and obesity among the studied students (Normal vs overweight \& obese).

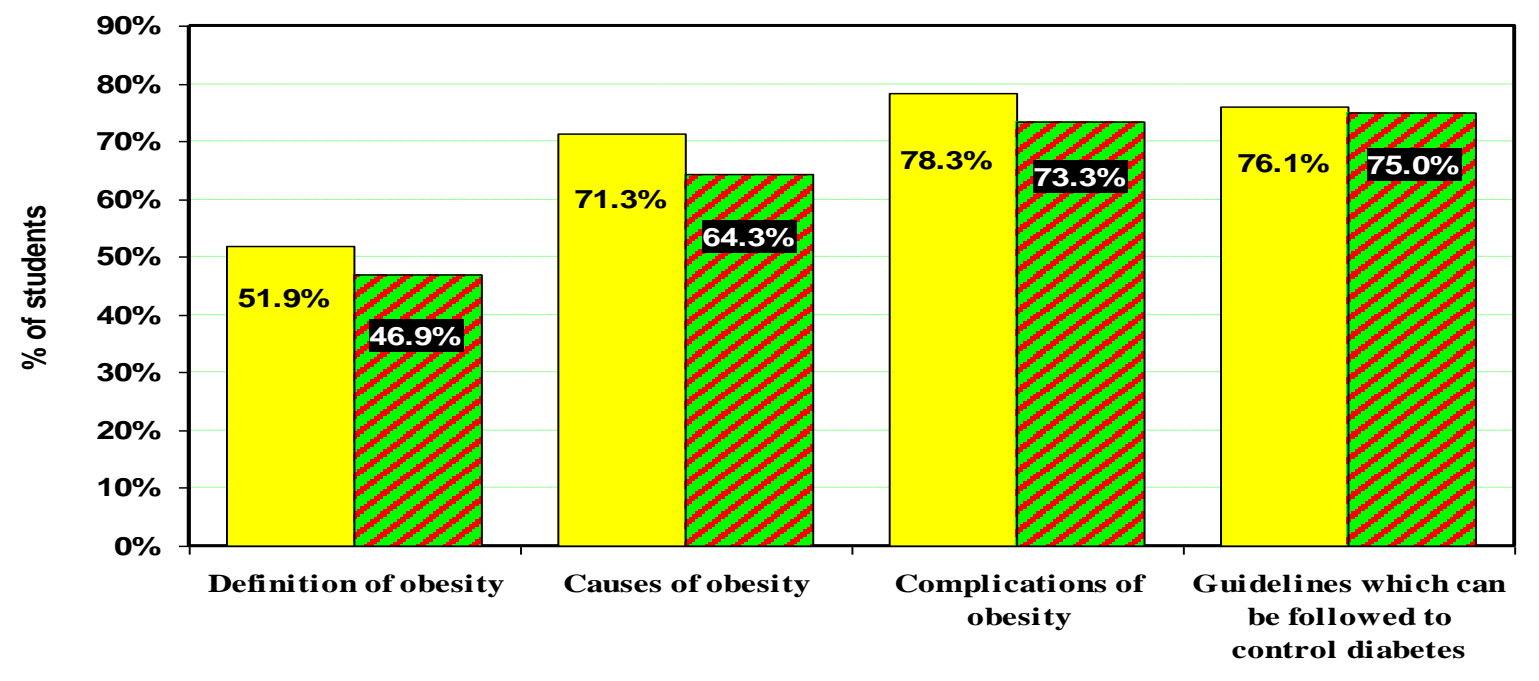

Correct information data about overweight \& obesity

Figure (2): Distribution of practicing physical exercise of the studied students (Normal vs overweight \& obese). 


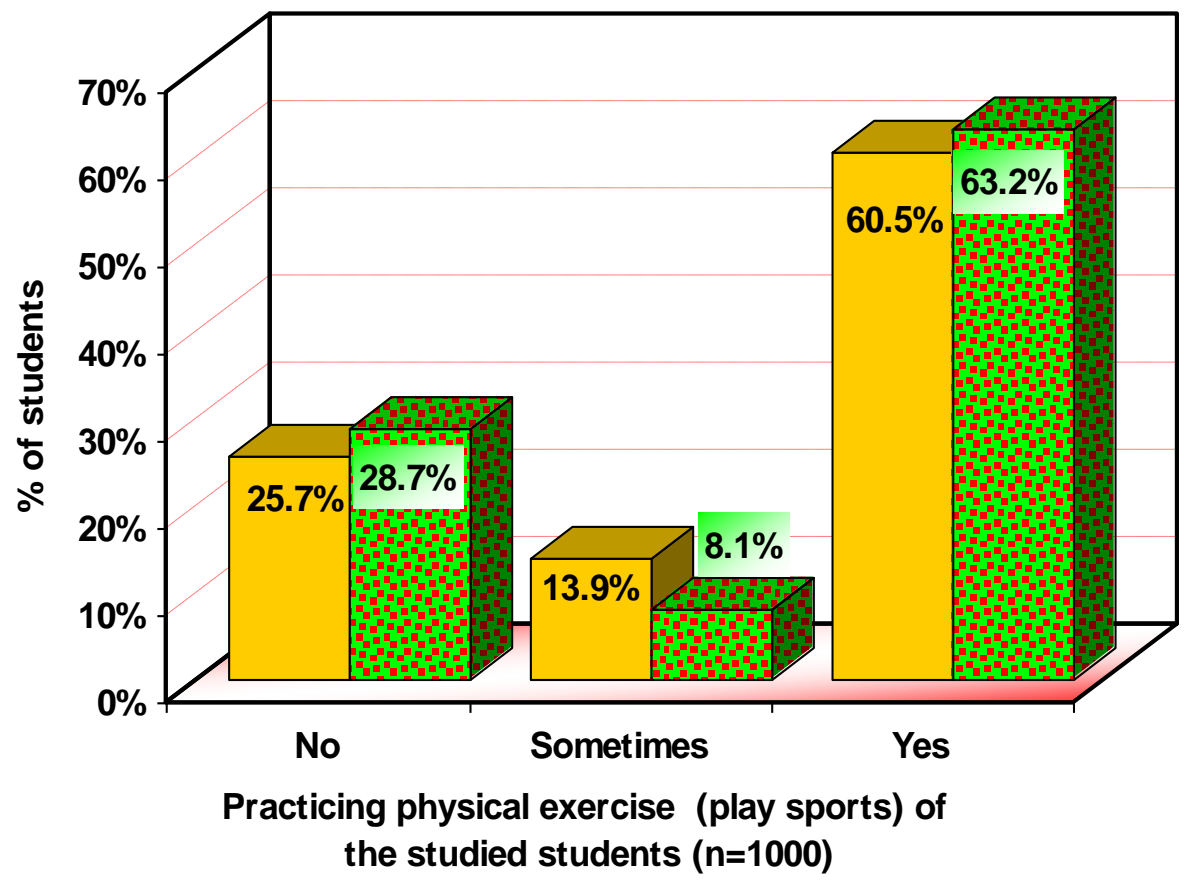

$\square$ Normal BW students ( $n=397)$ 回 Overweight \& Obese students $(n=603)$

Table (4):Percentage distribution of the studied students (Normal vs overweight \& obese) according to their dietary practices.

\begin{tabular}{|c|c|c|c|c|c|c|c|c|}
\hline \multirow{2}{*}{ Variables } & \multicolumn{2}{|c|}{$\begin{array}{c}\text { Normal body } \\
\text { weight students } \\
(n=397)\end{array}$} & \multicolumn{2}{|c|}{$\begin{array}{c}\text { Overweight \& } \\
\text { Obese students } \\
(n=603)\end{array}$} & \multicolumn{2}{|c|}{$\begin{array}{l}\text { Total students } \\
\quad(\mathbf{n}=\mathbf{1 0 0 0})\end{array}$} & \multirow[t]{2}{*}{$\chi^{2}$} & \multirow[t]{2}{*}{$\mathbf{P}$} \\
\hline & No & $\%$ & No & $\%$ & No & $\%$ & & \\
\hline $\begin{array}{l}\text {-Have information } \\
\text { about healthy diet: }\end{array}$ & & & & & & & & \\
\hline
\end{tabular}




\begin{tabular}{|c|c|c|c|c|c|c|c|c|}
\hline $\begin{array}{l}\text { No } \\
\text { Yes }\end{array}$ & $\begin{array}{l}102 \\
295\end{array}$ & $\begin{array}{l}25.7 \\
74.3\end{array}$ & $\begin{array}{l}133 \\
470\end{array}$ & $\begin{array}{l}22.1 \\
77.9\end{array}$ & $\begin{array}{l}235 \\
765 \\
\end{array}$ & $\begin{array}{l}23.5 \\
76.5\end{array}$ & 1.761 & 0.185 \\
\hline $\begin{array}{l}\text {-Follow the guidelines } \\
\text { about healthy diet : } \\
\text { No } \\
\text { Yes }\end{array}$ & $\begin{array}{l}121 \\
276\end{array}$ & $\begin{array}{l}30.5 \\
69.5\end{array}$ & $\begin{array}{l}188 \\
415\end{array}$ & $\begin{array}{l}31.2 \\
68.8\end{array}$ & $\begin{array}{l}309 \\
691\end{array}$ & $\begin{array}{l}30.9 \\
69.1\end{array}$ & 0.785 & 0.675 \\
\hline $\begin{array}{l}\text {-Number of meals/day: } \\
1-2 \\
3-4 \\
5 \& \text { more }\end{array}$ & $\begin{array}{c}174 \\
217 \\
6\end{array}$ & $\begin{array}{l}43.8 \\
54.7 \\
1.5\end{array}$ & $\begin{array}{c}238 \\
364 \\
1\end{array}$ & $\begin{array}{c}39.5 \\
60.4 \\
0.2 \\
\end{array}$ & $\begin{array}{c}412 \\
581 \\
7\end{array}$ & $\begin{array}{c}3.1 \\
54.7 \\
0.7\end{array}$ & 8.644 & $0.013^{*}$ \\
\hline $\begin{array}{l}\text {-Where you eat meals: } \\
\text { At home } \\
\text { Outside the home } \\
\text { Both }\end{array}$ & $\begin{array}{c}201 \\
8 \\
188 \\
\end{array}$ & $\begin{array}{c}50.6 \\
2.0 \\
47.4 \\
\end{array}$ & $\begin{array}{c}302 \\
14 \\
287\end{array}$ & $\begin{array}{c}50.1 \\
2.3 \\
47.6 \\
\end{array}$ & $\begin{array}{c}503 \\
22 \\
475\end{array}$ & $\begin{array}{c}50.3 \\
2.2 \\
47.5\end{array}$ & 0.119 & 0.942 \\
\hline •Eat between meals: & & & & & & & & \\
\hline $\begin{array}{l}\text { No } \\
\text { Yes } \\
\text { - Method of cooking } \\
\text { food: }\end{array}$ & $\begin{array}{l}145 \\
252\end{array}$ & $\begin{array}{l}36.5 \\
63.5\end{array}$ & $\begin{array}{l}199 \\
404\end{array}$ & $\begin{array}{l}33 \\
67\end{array}$ & $\begin{array}{l}344 \\
656\end{array}$ & $\begin{array}{l}34.4 \\
65.6\end{array}$ & 1.401 & 0.237 \\
\hline Boiled & 135 & 34.0 & 207 & 34.3 & 342 & 34.2 & 0.011 & 0.916 \\
\hline Grilled & 122 & 30.7 & 192 & 31.8 & 314 & 31.4 & 0.137 & 0.711 \\
\hline Foundry & 261 & 65.7 & 391 & 64.8 & 652 & 65.2 & 0.086 & 0.770 \\
\hline $\begin{array}{l}\text { Fried } \\
\text { What kind of fat used: }\end{array}$ & 171 & 43.1 & 280 & 46.4 & 451 & 45.1 & 1.092 & 0.296 \\
\hline Butter & 196 & 61.4 & 246 & 51.2 & 442 & 55.3 & 7.65 & $0.006^{*}$ \\
\hline Margarine & 92 & 28.8 & 162 & 33.7 & 254 & 31.8 & 1.91 & 0.167 \\
\hline Animal fat & 8 & 2.5 & 18 & 3.7 & 26 & 3.2 & 4.62 & $0.032 *$ \\
\hline Oil & 176 & 55.2 & 276 & 57.5 & 452 & 56.6 & 0.33 & 0.564 \\
\hline $\begin{array}{l}\text {-Eat vegetables with } \\
\text { meals: }\end{array}$ & & & & & & & & \\
\hline No & 84 & 21.2 & 138 & 22.9 & 222 & 22.2 & 1.991 & 0.370 \\
\hline $\begin{array}{l}\text { Yes } \\
\text {-Do you eat a lot of }\end{array}$ & 313 & 78.8 & 465 & 77.1 & 778 & 77.8 & & \\
\hline No & 236 & 59.4 & 364 & 60.4 & 600 & 60.0 & 0.408 & 0.815 \\
\hline Yes & 161 & 40.6 & 239 & 39.6 & 400 & 40.0 & & \\
\hline -Kind of daily fluid: & 319 & 803 & 368 & 610 & 787 & 787 & 4068 & $00001 *$ \\
\hline Soft water & 91 & $\begin{array}{l}00.5 \\
22.9\end{array}$ & $\begin{array}{l}500 \\
132\end{array}$ & 21.9 & 223 & 22.3 & $\begin{array}{c}40.00 \\
0.09\end{array}$ & 0.760 \\
\hline Juices & 177 & 44.6 & 234 & 38.8 & 411 & 41.1 & 3.07 & 0.080 \\
\hline Tea & 141 & 35.5 & 283 & 46.9 & 424 & 42.4 & 12.31 & $0.0004 *$ \\
\hline
\end{tabular}

*Significant $(\mathbf{P}<0.05)$ 
Table (5): Percentage distribution of macronutrients/24 hours (average of 3 days recall) of the studied students (Normal vs overweight \& obese).

\begin{tabular}{|c|c|c|c|c|c|}
\hline \multirow{2}{*}{$\begin{array}{c}\text { Macronutrients } \\
\text { taken/24 hours }\end{array}$} & $\begin{array}{c}\text { Normal body } \\
\text { weight students } \\
(\mathrm{n}=397)\end{array}$ & Overweight & Obese students & \multirow[t]{2}{*}{ F-value } & \multirow[t]{2}{*}{$\mathbf{P}$} \\
\hline & $\begin{array}{c}\text { Range } \\
\text { Mean } \pm \text { SD }\end{array}$ & $\begin{array}{c}\text { Range } \\
\text { Mean } \pm \text { SD }\end{array}$ & $\begin{array}{c}\text { Range } \\
\text { Mean } \pm \text { SD }\end{array}$ & & \\
\hline Water & $\begin{array}{c}186.40-1400 \\
535.64 \pm 181.68 \\
\end{array}$ & $\begin{array}{c}235.40-1395.50 \\
687.53 \pm 212.59 \\
\end{array}$ & $\begin{array}{c}292.80-1555.30 \\
793.19 \pm 259.01 \\
\end{array}$ & 109.22 & $\begin{array}{c}0.0001 \\
*\end{array}$ \\
\hline Calories (kcal) & $\begin{array}{c}563-3199 \\
1416.48 \pm 411.64\end{array}$ & $\begin{array}{c}189-3258 \\
1824.72 \pm 487.40\end{array}$ & $\begin{array}{c}88.90-3658 \\
1975.43 \pm 487.40\end{array}$ & 122.42 & $\begin{array}{c}0.0001 \\
*\end{array}$ \\
\hline Calories RDA & $\begin{array}{c}28.20-159 \\
70.66 \pm 20.65\end{array}$ & $\begin{array}{l}28.20-162.90 \\
91.31 \pm 23.96\end{array}$ & $\begin{array}{c}46.80-182.90 \\
98.61 \pm 25.00\end{array}$ & 127.22 & $\begin{array}{c}0.0001 \\
*\end{array}$ \\
\hline Protein-A (gm) & $\begin{array}{c}0-225 \\
27.63 \pm 16.66\end{array}$ & $\begin{array}{c}0-225 \\
32.23 \pm 17.75\end{array}$ & $\begin{array}{l}0.20-113.80 \\
33.26 \pm 17.03\end{array}$ & 10.091 & $\begin{array}{c}0.0001 \\
*\end{array}$ \\
\hline Protein-P (gm) & $\begin{array}{c}3.90-59.20 \\
25.98 \pm 9.40\end{array}$ & $\begin{array}{c}4.20-68.00 \\
35.01 \pm 11.52\end{array}$ & $\begin{array}{c}0.90-68.80 \\
38.09 \pm 12.75\end{array}$ & 104.57 & $\begin{array}{c}0.0001 \\
*\end{array}$ \\
\hline $\begin{array}{l}\text { Total protein } \\
\text { (gm) }\end{array}$ & $\begin{array}{l}12.50-103.50 \\
53.15 \pm 16.29\end{array}$ & $\begin{array}{l}15.40-114.30 \\
66.88 \pm 18.40\end{array}$ & $\begin{array}{l}25.90-136.50 \\
71.41 \pm 19.16\end{array}$ & 91.833 & $\begin{array}{c}0.0001 \\
*\end{array}$ \\
\hline $\begin{array}{l}\text { Total protein } \\
\text { RDA }\end{array}$ & $\begin{array}{l}12.70-178.40 \\
91.20 \pm 27.93\end{array}$ & $\begin{array}{l}10.90-197.10 \\
114.54 \pm 32.19\end{array}$ & $\begin{array}{l}44.70-235.30 \\
122.89 \pm 32.75\end{array}$ & 90.981 & $\begin{array}{c}0.0001 \\
*\end{array}$ \\
\hline Fat-A (gm) & $\begin{array}{c}0-145.30 \\
23.79 \pm 16.56\end{array}$ & $\begin{array}{c}0-128.40 \\
27.66 \pm 17.02\end{array}$ & $\begin{array}{c}0.10-72.40 \\
26.68 \pm 14.24\end{array}$ & 5.906 & $0.003^{*}$ \\
\hline Fat-P (gm) & $\begin{array}{c}0.60-113 \\
25.79 \pm 14.43\end{array}$ & $\begin{array}{c}0.60-123.40 \\
34.16 \pm 18.52\end{array}$ & $\begin{array}{c}0.60-92.30 \\
37.50 \pm 17.59\end{array}$ & 40.346 & $\begin{array}{c}0.0001 \\
*\end{array}$ \\
\hline Total fat (gm) & $\begin{array}{l}14.20-151.90 \\
48.77 \pm 19.72\end{array}$ & $\begin{array}{l}12.90-140.80 \\
61.30 \pm 22.66\end{array}$ & $\begin{array}{c}21-131.70 \\
64.45 \pm 21.40\end{array}$ & 49.921 & $\begin{array}{c}0.0001 \\
*\end{array}$ \\
\hline $\begin{array}{l}\text { Carbohydrate } \\
\text { (gm) }\end{array}$ & $\begin{array}{l}30.70-1175.70 \\
196.59 \pm 107.40\end{array}$ & $\begin{array}{l}30.70-442.30 \\
249.97 \pm 73.16\end{array}$ & $\begin{array}{l}109.70-501.60 \\
277.12 \pm 82.85\end{array}$ & 63.004 & $\begin{array}{c}0.0001 \\
*\end{array}$ \\
\hline Fiber (gm) & $\begin{array}{c}0.10-39.70 \\
14.71 \pm 6.15\end{array}$ & $\begin{array}{c}0.10-43 \\
19.50 \pm 7.58\end{array}$ & $\begin{array}{c}1.30-72.20 \\
21.12 \pm 8.70\end{array}$ & 66.622 & $\begin{array}{c}0.0001 \\
*\end{array}$ \\
\hline
\end{tabular}

*Significant $(\mathbf{P}<\mathbf{0 . 0 5})$

Table (6): Percentage Distribution of perception of personal factors that lead to obesity of the studied students (Normal vs overweight $\&$ obese). 


\begin{tabular}{|c|c|c|c|c|c|c|c|c|}
\hline & \multicolumn{2}{|c|}{$\begin{array}{c}\text { weight students } \\
(n=397)\end{array}$} & \multicolumn{2}{|c|}{$\begin{array}{l}\text { Obese students } \\
(n=603)\end{array}$} & \multicolumn{2}{|c|}{$(\mathrm{n}=1000)$} & & \\
\hline & No & $\%$ & No & $\%$ & No & $\%$ & & \\
\hline $\begin{array}{l}\text { - Information about } \\
\text { importance of physical } \\
\text { activity: }\end{array}$ & & & & & & & \multirow{3}{*}{15.751} & \\
\hline No & 154 & 48.7 & 162 & 51.3 & 316 & 31.6 & & \multirow[t]{2}{*}{$0.0001 *$} \\
\hline Yes & 243 & 51.3 & 441 & 48.7 & 684 & 68.4 & & \\
\hline $\begin{array}{l}\text { Source of information: } \\
\text {-General information \& } \\
\text { Newspapers }\end{array}$ & 41 & 16.9 & 120 & 27.2 & 161 & 23.5 & \multirow[t]{4}{*}{15.162} & \multirow[t]{4}{*}{$0.002 *$} \\
\hline -University \& Doctor & 87 & 35.8 & 127 & 28.8 & 214 & 31.3 & & \\
\hline -Friends \& relatives & 20 & 8.2 & 17 & 3.8 & 37 & 5.4 & & \\
\hline -Internet \& TV programs & 95 & 39.1 & 177 & 40.1 & 272 & 39.8 & & \\
\hline \multicolumn{9}{|l|}{$\begin{array}{l}\text { - Motivation to do physical } \\
\text { activity: }\end{array}$} \\
\hline No & 155 & 39.0 & 207 & 34.3 & 362 & 36.2 & \multirow[t]{2}{*}{2.304} & \multirow[t]{2}{*}{0.129} \\
\hline Yes & 242 & 61.0 & 396 & 65.7 & 638 & 63.8 & & \\
\hline \multicolumn{9}{|l|}{$\begin{array}{l}\text { - Enjoy for practicing } \\
\text { physical activitv: }\end{array}$} \\
\hline No & 138 & 34.8 & 197 & 32.7 & 335 & 33.5 & \multirow[t]{2}{*}{0.634} & \multirow[t]{2}{*}{0.426} \\
\hline Yes & 259 & 65.2 & 406 & 67.3 & 665 & 66.5 & & \\
\hline \multicolumn{9}{|l|}{ - Motivation to follow a } \\
\hline No & 61 & 15.4 & 117 & 19.4 & 178 & 17.7 & \multirow[t]{2}{*}{2.964} & \multirow[t]{2}{*}{0.085} \\
\hline Yes & 336 & 84.6 & 486 & 80.6 & 822 & 82.3 & & \\
\hline \multicolumn{9}{|l|}{ - Skill required to prepare } \\
\hline No & 77 & 19.4 & 120 & 19.9 & 197 & 19.7 & \multirow[t]{2}{*}{0.039} & \multirow[t]{2}{*}{0.844} \\
\hline Yes & 320 & 80.6 & 483 & 80.1 & 803 & 80.3 & & \\
\hline \multicolumn{9}{|l|}{$\begin{array}{l}\text {-Think that their weight is } \\
\text { appropriate for their height }\end{array}$} \\
\hline No & 157 & 39.5 & 366 & 60.7 & 523 & 52.3 & \multirow[t]{2}{*}{42.924} & \multirow[t]{2}{*}{$0.0001 *$} \\
\hline Yes & 240 & 60.5 & 237 & 39.3 & 477 & 47.7 & & \\
\hline $\begin{array}{l}\text {-Weigh yourself regularly: } \\
\text { No }\end{array}$ & 226 & 56.9 & 352 & 58.4 & 578 & 57.8 & 0.206 & 0.650 \\
\hline Yes & 171 & 43.1 & 251 & 41.6 & 422 & 42.2 & & \\
\hline \multicolumn{9}{|l|}{$\begin{array}{l}\text { - Satisfied with your body } \\
\text { weight: }\end{array}$} \\
\hline Not satisfied & 231 & 58.2 & 261 & 43.3 & 492 & 49.2 & \multirow[t]{3}{*}{21.554} & \multirow[t]{3}{*}{$0.0001 *$} \\
\hline Satisfied & 164 & 41.3 & 339 & 56.2 & 503 & 50.3 & & \\
\hline Somewhat & 2 & 0.5 & 3 & 0.5 & 5 & 0.5 & & \\
\hline $\begin{array}{l}\text { - problems with body weight: } \\
\text { No }\end{array}$ & 242 & 61.0 & 319 & 52.9 & 561 & 56.1 & \multirow[t]{2}{*}{6.307} & \multirow[t]{2}{*}{$0.012^{*}$} \\
\hline Yes & 155 & 39.0 & 284 & 47.1 & 439 & 43.9 & & \\
\hline
\end{tabular}

*Significant $(\mathbf{P}<\mathbf{0 . 0 5})$

Table (7): Percentage Distribution of perception of environmental factors that lead to obesity of the studied students (Normal vs overweight \& obese).

\begin{tabular}{|c|c|c|c|c|c|c|c|c|}
\hline \multirow[t]{2}{*}{$\begin{array}{l}\text { Perceived environmental } \\
\text { factors }\end{array}$} & \multicolumn{2}{|c|}{$\begin{array}{c}\text { Normal body } \\
\text { weight students } \\
(\mathrm{n}=397)\end{array}$} & \multicolumn{2}{|c|}{$\begin{array}{c}\text { Overweight \& } \\
\text { Obese students } \\
(n=603)\end{array}$} & \multicolumn{2}{|c|}{ Total students } & \multirow[t]{2}{*}{$\chi^{2}$} & \multirow[t]{2}{*}{$\mathbf{P}$} \\
\hline & No & $\%$ & No & $\%$ & No & $\%$ & & \\
\hline $\begin{array}{l}\text {-Have time for physical } \\
\text { activity: }\end{array}$ & & & & & & & & \\
\hline
\end{tabular}




\begin{tabular}{|c|c|c|c|c|c|c|c|c|}
\hline $\begin{array}{l}\text { No } \\
\text { Yes }\end{array}$ & $\begin{array}{l}240 \\
157\end{array}$ & $\begin{array}{l}60.5 \\
39.5\end{array}$ & $\begin{array}{l}361 \\
242\end{array}$ & $\begin{array}{l}59.9 \\
40.1\end{array}$ & $\begin{array}{l}601 \\
399\end{array}$ & $\begin{array}{l}60.1 \\
39.9\end{array}$ & 0.034 & 0.853 \\
\hline $\begin{array}{l}\text {-There is a safe environment } \\
\text { for physical activity: } \\
\text { No } \\
\text { Yes }\end{array}$ & $\begin{array}{l}225 \\
172 \\
\end{array}$ & $\begin{array}{l}56.7 \\
43.3\end{array}$ & $\begin{array}{l}341 \\
262 \\
\end{array}$ & $\begin{array}{l}56.6 \\
43.4\end{array}$ & $\begin{array}{l}566 \\
434 \\
\end{array}$ & $\begin{array}{r}56.6 \\
43.4 \\
\end{array}$ & 0.002 & 0.969 \\
\hline $\begin{array}{l}\text {-Have a way to access to the } \\
\text { places for physical activity: } \\
\text { No } \\
\text { Yes }\end{array}$ & $\begin{array}{l}238 \\
159 \\
\end{array}$ & $\begin{array}{l}59.9 \\
40.1 \\
\end{array}$ & $\begin{array}{l}389 \\
214 \\
\end{array}$ & $\begin{array}{l}64.5 \\
35.5 \\
\end{array}$ & $\begin{array}{l}627 \\
373 \\
\end{array}$ & $\begin{array}{l}62.7 \\
37.3 \\
\end{array}$ & 2.130 & 0.144 \\
\hline $\begin{array}{l}\text {-Have time to prepare or eat } \\
\text { healthy foods: } \\
\text { No } \\
\text { Yes }\end{array}$ & $\begin{array}{l}155 \\
242 \\
\end{array}$ & $\begin{array}{l}39.0 \\
61.0 \\
\end{array}$ & $\begin{array}{l}268 \\
335 \\
\end{array}$ & $\begin{array}{l}44.4 \\
55.6\end{array}$ & $\begin{array}{l}423 \\
577 \\
\end{array}$ & $\begin{array}{l}42.3 \\
57.7 \\
\end{array}$ & 2.862 & 0.091 \\
\hline
\end{tabular}

*Significant $(\mathbf{P}<0.05)$

Table (8): Precentage distribution of perception of social factors that lead to obesity of the studied students (Normal vs overweight \& obese).

\begin{tabular}{|c|c|c|c|c|c|c|c|c|}
\hline \multirow[t]{2}{*}{ Perceived social factors } & \multicolumn{2}{|c|}{$\begin{array}{c}\text { Normal body } \\
\text { weight students } \\
(n=397)\end{array}$} & \multicolumn{2}{|c|}{$\begin{array}{c}\text { Overweight \& } \\
\text { Obese students } \\
(n=603)\end{array}$} & \multicolumn{2}{|c|}{$\begin{array}{l}\text { Total students } \\
\qquad(\mathbf{n}=\mathbf{1 0 0 0})\end{array}$} & \multirow[t]{2}{*}{$\chi^{2}$} & \multirow[t]{2}{*}{$\mathbf{P}$} \\
\hline & No & $\%$ & No & $\%$ & No & $\%$ & & \\
\hline $\begin{array}{l}\text {-There is support from the } \\
\text { spouse or family members for } \\
\text { physical activity: } \\
\text { No } \\
\text { Yes }\end{array}$ & $\begin{array}{l}281 \\
116 \\
\end{array}$ & $\begin{array}{r}70.8 \\
29.2 \\
\end{array}$ & $\begin{array}{l}387 \\
216 \\
\end{array}$ & $\begin{array}{r}64.2 \\
35.8 \\
\end{array}$ & $\begin{array}{l}668 \\
332 \\
\end{array}$ & $\begin{array}{l}66.8 \\
33.2 \\
\end{array}$ & 4.704 & $0.030 *$ \\
\hline $\begin{array}{l}\text {-Have cost-effective places for } \\
\text { practicing physical activity: } \\
\text { No } \\
\text { Yes }\end{array}$ & $\begin{array}{l}290 \\
107\end{array}$ & $\begin{array}{r}73.0 \\
27.0 \\
\end{array}$ & $\begin{array}{l}425 \\
178\end{array}$ & $\begin{array}{r}70.5 \\
29.5 \\
\end{array}$ & $\begin{array}{l}715 \\
285 \\
\end{array}$ & $\begin{array}{l}71.5 \\
28.5 \\
\end{array}$ & 0.732 & 0.392 \\
\hline $\begin{array}{l}\text {-There is a support from the } \\
\text { spouse or family members to } \\
\text { follow a healthy diet: } \\
\text { No } \\
\text { Yes }\end{array}$ & $\begin{array}{l}165 \\
232 \\
\end{array}$ & $\begin{array}{l}41.6 \\
58.4 \\
\end{array}$ & $\begin{array}{l}288 \\
315 \\
\end{array}$ & $\begin{array}{l}47.8 \\
52.2 \\
\end{array}$ & $\begin{array}{l}453 \\
547 \\
\end{array}$ & $\begin{array}{l}45.3 \\
54.7 \\
\end{array}$ & 3.713 & 0.054 \\
\hline $\begin{array}{l}\text {-Healthy food expensive: } \\
\text { No } \\
\text { Yes }\end{array}$ & $\begin{array}{l}204 \\
193\end{array}$ & $\begin{array}{l}51.4 \\
48.6\end{array}$ & $\begin{array}{l}277 \\
326 \\
\end{array}$ & $\begin{array}{l}45.9 \\
54.1\end{array}$ & $\begin{array}{l}481 \\
519\end{array}$ & $\begin{array}{l}48.1 \\
51.9\end{array}$ & 2.847 & 0.092 \\
\hline
\end{tabular}

*Significant $(\mathbf{P}<\mathbf{0 . 0 5})$

\section{Discussion}

Obesity prevalence among adolescents is increasing world wide and may be associated risk for development of chronic diseases in adulthood. Dissatisfaction with weight and body shapes is a contributor to the growing incidence of adolescent eating disorders (Jackson et al., 2003).

\section{Medical History}

Regarding student's family history of overweight and obesity the result of the present study showed that one third of the overweight and obese students had positive family history of overweight. This is in agreement with Hassan, (2006) who found 
that the risk of developing overweight and obesity among the subjects who had positive family history of overweight and obesity were higher than among those who had negative family history. This could be because family history of obesity on one hand may reflect the genetic predisposition for obesity, and on the other hand it may reflect behaviors in the family that may lead to sedentary unhealthy lifestyle.

As regard anthropometric measurements, the results of this study clarified that, more than half of the students were overweight and obese. In the same line with the present finding was the study carried out by Moldenhauer et al., (2002) found that $85 \%$ of college females' students believed that they were either slightly or seriously overweight and $5 \%$ of college female students below their ideal weight. In contradicting with the study, Najat et al., (2008) reported that the majority of students were of normal weight (76.8\%). And Hoda et al., (2010) found that the minority of the participants $(7.8 \%)$ considered them obese.

\section{Regarding student's knowledge about}

obesity, the current study revealed that the majority of normal body weight students and overweight and obese students knew the correct answer about complication of obesity. Also, the results of Rehman et al.,
(2003) in a study done to investigate obesity of adolescent in Pakistan was in agreement with the results of the present study as they found that the majority of the sample knew the harmful effects of overweight.

Also, the results clarified that the majority of both normal body weight vs overweight and obese students knew the correct answers about causes of obesity, complication and guidelines which can be followed to control obesity. This result was not supported by Hassan, (2006) who found that overweight and obese sample had satisfactory mean score of knowledge while the sample of normal weight had good mean score of knowledge. It could be because the students of normal weight who had good knowledge can maintain their body weight. Although, the obese and overweight had high knowledge score about causes, complication of obesity and guidelines which can be followed to control obesity, this knowledge did not change their behavior of food habits, in turn they are more liable to develop overweight and obesity.

\section{Practicing physical activity}

As for practicing physical activity the current study revealed that the majority of the students were practicing exercises. This result was higher than the results of Coaccioli et al., (2006) who found that 
approximately $41 \%$ of adolescents were practicing exercise. This could be because walking to university is considered exercise for the current students.

\section{Dietary practice}

According to the information about healthy diet, the present study reported that more than two thirds of students in both groups have information about healthy diet. This was supported by Najat et al., (2008) who found that $73 \%$ female students in these study agreed that it is important to eat a variety of foods to have a balanced and nutritious diet. Also a study conducted at Midwestern University among 105 male and 181 female students reported that $94.4 \%$ of the students agreed that it is important to eat a variety of foods for good health (Davy et al., 2006). Also Kolodinsky et al., (2007) reported that increased knowledge of dietary guidance, Dietary Guidelines for Americans 2005, appeared to be positively related to healthier eating patterns thus the better eaters had a higher level of knowledge about nutrition. This finding could be because university educations equip students with health information. So, the normal weight students maintain their weight, while overweight and obese are always seeking knowledge to control their weight.

According to number of meals, the present study showed that less than two thirds of students eat three to four meals per day. This result was supported by Hassan, (2006) who found that the percentage of obese sample who consumed 4 meals or more per day was higher than those of the non obese sample. This finding was contradicting with Pon et al., (2004) who found that students practiced a consistent mealtime for their daily meal consumption, $36 \%$ of the subjects responded yes, 38\% responded sometimes while $26 \%$ said that they did not.

Concerning eating between meals, the current study clarified that more than two thirds of students reported eating snacks. This was supported by Najat et al., (2008) who found daily intake of snacks a part from regular meals. Also Pon et al., (2004) found that more girls in both groups reported they eating snacking in between meals. Group comparison of this habit, however, failed to show a significant difference. This could be due to eating at the university at free time.

According to dietary recall, the present 
study revealed significant increase is fiber intake among overweight \& obese students than normal weight. This result in contradicting with Strong et al., (2008) who found that the majority of college students do not consume enough fiber. Contradicting with the present study Walters, (2009) who reported that BMI was not significantly correlated with reported fiber consumption $(\mathrm{r}=0.582 ; \mathrm{p}>0.05)$.

According to the present study, the results revealed that there was significant increase in protein consumption among overweight $\&$ obese students than normal weight. This finding revealed that overweight and obese students consumed more food that is rich in every thing (fiber, fat, and calcium) more frequently and larger portion of food than those of normal weight. This finding was supported by Murray \& Vickers, (2009) who reported that consumption of foods high in protein may increase satiety, which results in fewer calories consumed. This may be due to protein-induced satiety appears to be of vital importance for weight loss and weight maintenance. Contradicting with the present study Walters, (2009) reported that BMI was not significantly correlated with reported protein consumption $(r=0.478 ; \mathrm{p}>0.05)$.
Also, the present study reported that there was significant difference between normal, overweight \& obese students regarding calcium and calcium recommended dietary allowance (RDA). This finding is incongruent with Walters, (2009) who found that BMI was not significantly correlated with reported calcium consumption $(r=0.061 ; p>0.05)$.

Concerning calories consumption, the present study reported that there was significant difference between normal, overweight \& obese students regarding calories. This result is incongruent with Walters, (2009) who reported that BMI was not significantly correlated with reported calorie consumption $(\mathrm{r}=0.375 ; \mathrm{p}>0.05)$. This may be due to overweight individuals tend to either underestimate the amount of food consumed, or be in denial about how much food they are consuming.

Regarding carbohydrate consumption, the present study reported that carbohydrate intake by overweight and obese students were higher than normal body weight students. This result is incongruent with Rose et al., (2007) who mentioned that whole-grain intake in college students of normal weight was significantly higher than whole-grain consumption in overweight or obese students. 
As for sodium consumption, the present study revealed that there was significant difference between normal, overweight and obese students regarding sodium and sodium RDA. This finding is supported by Larson et al., (2009) who found that college students consume excessive amounts of high-sodium foods. This may be due to high level of sodium consumed from fast food, which college students are notorious for consuming in extreme amounts.

Personal factors leading to obesity: Regarding information about importance of physical activity, the finding represented that more than half of the students of the two faculties' had information about importance of physical activity. This result was supported by Samir et al., (2011) who mentioned that among inactive participants more than a quarter admitted that they had information regarding importance of physical activity. Also, Andajani-Sutjahjo etal, (2004).found that lack of information and skills were less common perceived barriers to physical activity.

As regard to motivation and enjoying doing physical activity, the finding of the current study found that about two thirds of overweight and obese students had motivation and enjoying doing physical activity. This is incongruent with Samir et al., (2011) mentioned that lack of motivation to do physical activity and not enjoying physical activity was reported by more than half of obese inactive attendants, whereas more than three-fourths reported that they did not have skills for physical activity. Also, Andajani-Sutjahjo etal, (2004) found that lack of motivation to do physical activity.

According to skills to prepare healthy foods, the findings of the present study revealed that the majority of normal vs overweight and obese students were motivated to follow a healthy diet instruction and have the skill required to prepare healthy foods. This result was in contradicting with Andajani-Sutjahjo etal, (2004) and AL Farwan, (2011) who mentioned that the main barriers reported by women related to a need for special prepared, separated food from family, accounting for $62.3 \%$, lack of information, skills to plan and prepare for a healthy diet accounting for $61.2 \%$, easy availability of fast food (56\%), lack of motivation to eat healthy diet accounting for $56.6 \%$,

\section{Environmental factors lead to obesity}

In relation to time for physical activity, this finding of the current study found that more than half of the students of the two faculties' had lack of time for physical 
activity. This result was supported by Daskapan et al., (2006) who reported that the greatest barrier was time constraint due to school work, social and family activities on high school students. Also, AndajaniSutjahjo etal, (2004) found lack of time due to family commitments $(37 \%)$, perhaps due to the relatively small proportion (30\%) of young women in this study with at least one child. This may be due to busy lesson schedule, parents give academic success priority over exercise, have no leisure time for exercise because of social and family responsibilities.

According to safe environment for physical activity, the present study showed that the majority of overweight, obese and normal students had lack of safe environment, lack of accessibility to places for physical activity. This result was supported by Andajani-Sutjahjo etal, (2004) and Samir et al., (2011) who mentioned that lack of access to places for physical activity, and lack of safe outdoor environment to some extent were found to be important environmental barriers among inactive obese individuals. This might be due to lack of recreational place for exercising in rural area.

\section{Social factors lead to obesity}

\section{Regarding support form spouse or family} members for physical activity, the current study reported that more than two thirds of students reported absence of spouse or family support for physical activity and lack of cost affordable facilities. This result was in the same line with Andajani-Sutjahjo etal, (2004) and Samir et al., (2011) who mentioned that social barriers about half of the individuals reported absence of spouse and children's support for physical activity, while more than half reported absence of other family member's support for physical activity.

As for healthy food expenses; of the present study clarified more that half of the obese \&overweight students reported that the healthy food is expensive. This result comes in agreement with AndajaniSutjahjo etal, (2004) and AL Farwan, (2011) who reported that cost of healthy food accounting for $48 \%$ and the perception that healthy foods are inconvenient or expensive (13\%). This may be because this group may regard the cost of food as prohibitive to eating a more healthy diet. Thus, it is necessary to ensure that foods that are included in a healthy diet are not too expensive or perceived as such.

\section{Conclusions}


Based on the results of this study, it can be concluded that, lack of time, no safe environment and lack of access to the places for exercising, absence of spouse or family support for physical activity and lack of cost affordable facilities and unhealthy diet lead to obesity.

\section{Recommendations}

Based on the findings of the present study the following recommendations are derived and suggested for future researches:-

\section{Recommendation related to home environment:}

1. Parents should be a role model by practicing healthy eating and exercise habit for their teenagers to promote a healthy eating and lifestyle.

\section{Recommendation for national} government:

1. Community attention should be focused on counseling services for the health related issues such as nutrition and dietary practices.

2. Ministry of Education and Ministry of Health should initiate national strategies for health education of adolescents based on family life and healthy life style including dietary practices and nutrition.

Recommendation for future researches:
Future research should study further psychosocial stress and educational environment stress that influence food intake, selection, and preparation, attitude toward health, fitness and activity among female students in Menoufia University

\section{Reference:}

1. Adams, J., and Murphy, Obesity in anesthesia and intensive care. $\mathrm{Br} \mathrm{J}$ Anaesth, 2006; 85 (1); 91-108.

2. AL Farwan Wadha Mushabeb, Perceived personal, social, and environmental barriers to healthy eating among young overweight and obese Saudi women. Middle East Journal of Family Medicine; 2011; 9 (10):3

3. Andajani-sutahjo,S.; Ball, K.; Warren, N.; Inglis, V., and Crawford, D Perceived personal,Social and environmental barriers to weight maintantenance among young women: Acommunity survey. The International Journal of Behavioral Nutrition Physical Activity, 2004; 1(1): 15.

4. Ball, K.; Crawford, D., and Warren, N. How feasible are healthy eating and physical activity for young women? Public Health Nutr, 2004; 7 (3): 433441. 
5. Barness, L.; Opitz, J., and Gilbert-

Barness, E. Obesity: Genetic, molecular and environmental aspects. Am J Med

Genet, 2007; 143 (24): 3016-34.

6. Brownson, R.C.; Baker, E.A.; Housemann, R.A.;, Brennan, L.K., and Bacak, S.J. Environmental and policy determinants of physical activities in the United States. American Journal of Public Health, 200; 91 (12):1995-2003

7. Central Agency for Public Mobilization and Statistics (CAPMAS). The State of Food Security and Vulnerability in Egypt. Draft (unpublished). April 2011.

8. COACCIOLI, S.; PONTEGGIA, M.; PONTEGGIA, F.; FATATI, G., DI GIANVITO, A., and PUXEDDU, A. Osteoporosis prevention: A reasoned examination of food habits and physical activities in a schoolchildren population in central Italy. Clin. Ter, 2006; 157 (6): 489-94.

9. Daskapan Arzu; Emine Handan Tuzun and Levent Eker . (2006). PERCEIVED BARRIERS TO PHYSICAL ACTIVITY IN UNIVERSITY STUDENTS. Journal of Sports Science and Medicine 5, 615-620 .Available at:
- http://www.jssm.org Retrieved on, 2013.

10. Davy, S.; Benes, B., and Driskell, J. Sex Differences in Dieting Trends, Eating Habits, and Nutrition Beliefs of a Group of Midwestern College Students. Journal of the American Dietetic Association,2006; 106(10): 1673-1677.

11. Hassan, Lulah. Abd-El Wahab. Abd- El Aty, Investigate Factors Cotributing to Overweight among Adolscents in Tanta City, MCS, Community Health Nursing, Tanta University , 2006; , $25-45,170-180$.

12. HODA D.F. IBRAHIM; et al., Body Image, Eating Habits and Practice Exercises Attitudes of Female Adolescent Students at Assiut University. Med. J. Cairo Univ, 2010; 78(2): 765-772.

13. Hossain, P.; Kawar, B., and El Nahas, M. Obesity and Diabetes in the Developing World - A Growing Challenge. New England Journal of Medicine, 2007; 356:213-215 .

14. International Council of Nurses. ICN on obesity: creating public awareness of a social-environmental disease. ICN Fact sheet. Retrieved on (May 20, 2012), Available at: http://www.icn.ch/images/stor 
ies/documents/publications/fact_sheets/ 13d_FS-Obesity.pdf

15. Jackson, R. The impact of the built environment on health: an emerging field. Am J Public Health, 2003; 93(9); 1382-1384

16. Kear ME, Bear M.Using portfolio evaluation for program outcome assessment .J Nurse Educ; .2007; 46(3):109-14

17. Kolodinsky, J., Harvey-Berino, J.R., Berlin, L., Johnson, R.K., and Reynolds, T.W. Knowledge of Current Dietary Guidelines and Food Choice by College Students: Better Eaters Have Higher Knowledge of Dietary Guidance. J Am Diet Assoc, 2007; 107, 1409-1413.

18. Kopelman, P. Health risks associated with overweight and obesity. Obesity Reviews; 2007; 8 (1); 13-17.

19. Larson, N.; Nelson, M.; NeumarkSztainer, D.; Story, M., \& Hannan, P. Making time for meals: meal structure and associations with dietary intake in young adults. Journal of the American Dietetic Association, 2009;109, 72-79.

20. MOLDENHAUER, K.; CALLICUTT, A., and LEDDICK, G. Body image among female college students. Copyright EBSCO publishing, 2002; 155-61.
21. Murray, M., \& Vickers, Z. Consumer views of hunger and fullness. A qualitative approach. Appetite, 2009;10.

22. Najat Yahia1; Alice Achkar; Abbass Abdallah and Sandra Rizk. Eating habits and obesity among Lebanese university students Published: Nutrition Journal,2008; 7(32), 1- 6.

23. National Nutrient Database for standard Reference, Dietary reference intake: Vitamin A, vitamin $\mathrm{K}$, arsenic, born, chromium, copper, iodine, iron, manganese, molybdenum, nickel, silicon, vanadium, and zinc. 2000. Available at:http:"119.93.23.123/resourcematerials/ ACADEME/e20 guide\%20\%20nutrient\%20requirement. pdf. Retrieved on:- January 5, 2013.

24. Petrie $A$ and Sabin C. Medical Statistics at a Glance. 2nd ed., Blackwell Publishing. 2005.

25. Pon L W; KandiahM \& Mohd Nasir M T, Body Image Perception, Dietary Practices and Physical Activity of Overweight and Normal Weight Malaysian Female Adolescents. Mal J Nutr 2004;10(2): 131-147

26. Puoane, T.; Steyn, K.; Bradshaw, D.; Laubscher, R.; Fourie, J., Lambert, V., and Mbananga, N. Obesity in South 
Africa: the South African demographic and health survey. Obesity Research, 2002;.10, 1038-1348.

27. Rehman T; Rizvi Z;Siddiqui .; Ahmad S; Sophie A; Siddiqui M; Saeed O; Kizilblash Q; Lakhani A, and shakoor A Obesity in Adolscent of Pakistan. Journal of Pakistan Medical Association. 2003; (7):15-21

28. Rose, N.; Hosig, K.; Davy, B.; Serrano, E., \& Davis, L. Whole-grain intake is associated with body mass index in college students. Journal of Nutrition Education Behavior, 2007; 39 (2), 90-4.

29. Samir Nafisa; Sadia Mahmud and Ali Khan Khuwaja Prevalence of physical inactivity and barriers to physical activity among obese attendants at a community health-care center in Karachi, Pakistan. BMC Research Notes, 2011; 174(4), 1-7.

30. Sproston, K. and P. Primetesta Health Survey of England., the health of children and young people, The Stationery Office: London 2003.

31. Strong, K.; Parks, S.; Anderson, E.; Winett, R., \& Davy, B. Weight gain prevention: identifying theory-based targets for health behavior change in young adults. Journal of the American
Dietetic Association, 2008; 108 (10): 1708-1715.

32. Walters, L B. A descriptive study of the diet and physical activity practices of overweight and obese college students enrolled in a weight loss program" university of Kentucky master's. Theses. Paper 633. Available at:http://uknowledge.uky.edu/gradschool_t heses/633. Retrieved on: - (May 6, 2013.

33. Whitlock EP, O'Connor E, Williams SB, Beil TL, Lutz KW. Effectiveness of Weight Management in Children and Adolescents. Rockville, MD: Agency for Healthcare Research and Quality; AHRQ Publication 08-E014, Evidence Report/Technology Assessment 2008; 170

34. World Health Organization. .The AsiaPacific perspective: redefining obesity and its treatment. Health Communications Australia Pty Ltd.Availableat:http://www.idi.org.au/o besity-report.htm. Retrieved on: (January 6, 2013). 
